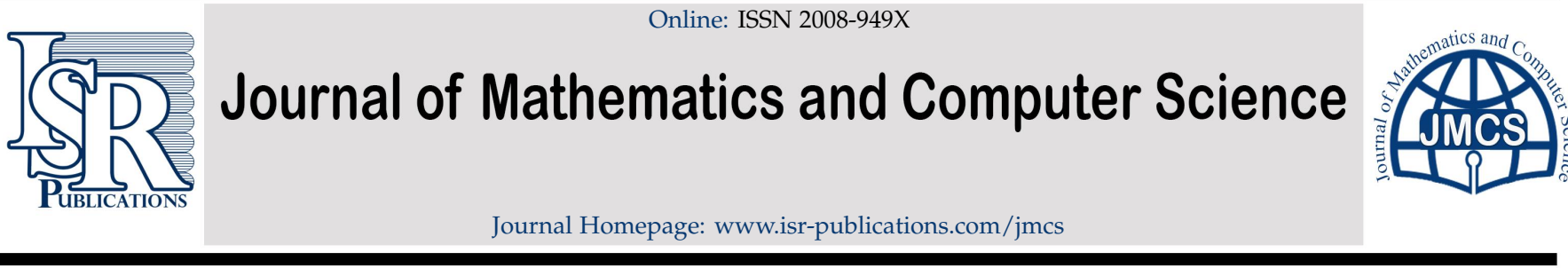

\title{
Stability of an additive-quartic functional equation in mod- ular spaces
}

\author{
S. Karthikeyan ${ }^{\mathrm{a}, *}$, Choonkil Park ${ }^{\mathrm{b}}$, P. Palanic, T. R. K. Kumar ${ }^{\mathrm{a}}$ \\ ${ }^{a}$ Department of Mathematics, R.M.K. Engineering College, Kavaraipettai - 601 206, Tamil Nadu, India. \\ ${ }^{b}$ Research Institute for Natural Sciences, Hanyang University, Seoul 04763, Korea. \\ ${ }^{c}$ Department of Mathematics, Sri Vidya Mandir Arts and Science College, Uthangarai- 636 902, Tamil Nadu, India.
}

\begin{abstract}
In this paper, we prove the Ulam-Hyers stability of the following additive-quartic functional equation

$$
\mathrm{f}\left(\frac{\mathrm{u}+v}{2}-w\right)+\mathrm{f}\left(\frac{v+w}{2}-\mathrm{u}\right)+\mathrm{f}\left(\frac{w+u}{2}-v\right)=\frac{25}{32}(\mathrm{f}(\mathrm{u}-v)+\mathrm{f}(v-w)+\mathrm{f}(w-u))-\frac{7}{32}(\mathrm{f}(v-\mathrm{u})+\mathrm{f}(w-v)+\mathrm{f}(\mathrm{u}-w))
$$
\end{abstract}

in modular spaces by using the direct method.

Keywords: Ulam-Hyers stability, additive functional equation, quartic functional equation, modular space.

2020 MSC: 39B52, 39B72, 39B82.

(C)2022 All rights reserved.

\section{Introduction}

Stability of the functional equation has been growing tremendously over the last seventy years. Ulam [43], who was responsible for this, raised the question of stability at a conference in 1940. The following year, a mathematician named Hyers [11] gave an answer based on Ulam's question. Aoki [2] published a paper in1950 generalizing Hyers' response. Then in 1978, Rassias [36] introduced a new stability result as sum of two norms. Rassias [37] converted to the multiplication of two norms in 1982. In 1994, Găvruta [9] provided a further generalization of Rassias theorem in which he replaced the bound by a general control function. In 2008, Ravi [40] established mixed type stability by adding sum of two norms and product of two norms. Subsequent authors have given flexible results using a lot of functional equations in modular spaces $[4,10,22,32,34,35,44,45]$.

The additive functional equation is

$$
f(x+y)=f(x)+f(y) .
$$

\footnotetext{
*Corresponding author

Email addresses: kns.sh@rmkec.ac.in (S. Karthikeyan), baak@hanyang.ac.kr (Choonkil Park), ppalanitamil@gmail.com (P.

Palani), trk.sh@rmkec.ac.in (T. R. K. Kumar)
}

doi: $10.22436 /$ jmcs.026.01.04

Received: 2021-07-10 Revised: 2021-07-21 Accepted: 2021-08-10 
Since $f(x)=k x$ is the solution of the functional equation (1.1), every solution of the additive functional equation is called an additive mapping. The functional equation

$$
f(2 x+y)+f(2 x-y)=4 f(x+y)+4 f(x-y)+24 f(x)-6 f(y)
$$

is called a quartic functional equation. Since the function $f(x)=x^{4}$ is a solution of (1.2), every solution of the quartic functional equation is called a quartic mapping.

In this paper, we present the Ulam-Hyers stability of the additive-quartic mixed type functional equation of the form

$$
\begin{aligned}
& f\left(\frac{u+v}{2}-w\right)+f\left(\frac{v+w}{2}-u\right)+f\left(\frac{w+u}{2}-v\right) \\
& \quad=\frac{25}{32}(f(u-v)+f(v-w)+f(w-u))-\frac{7}{32}(f(v-u)+f(w-v)+f(u-w))
\end{aligned}
$$

in modular spaces by using the direct method.

\section{Basic concepts on modular space}

The research on modulars and modular spaces was begun by Nakano [31] as generalizations of normed spaces. Since the 1950s, many prominent mathematicians like Luxemburg, Mazur, Musielak, and Orlicz [25, 26, 29, 30] developed it extensively. Modulars and modular spaces have broad branches of applications, e.g., interpolation theory and Orlicz spaces. We start by considering some basic relevant notions.

Definition 2.1. Let $X$ be a vector space over a field $\mathbb{K}(\mathbb{R}$ or $\mathbb{C})$. A generalized function $\rho: X \rightarrow[0, \infty]$ is called a modular if for any $\alpha, \beta \in \mathbb{K}$ and $x, y \in X$,

(1) $\rho(x)=0$ if and only if $x=0$;

(2) $\rho(\alpha x)=\rho(x)$ for every $\alpha$ with $|\alpha|=1$;

(3) $\rho(\alpha x+\beta y) \leqslant \rho(x)+\rho(y)$ if $\alpha+\beta=1$ and $\alpha, \beta \geqslant 0$.

If the condition (3) is replaced with

(4) $\rho(\alpha x+\beta y) \leqslant \alpha^{s} \rho(x)+\beta^{s} \rho(y)$ if $\alpha s+\beta s=1$ and $\alpha, \beta \geqslant 0$ with an $s \in(0,1]$,

then $\rho$ is called an $s$-convex modular. We call 1-convex modulars as convex modulars.

A modular $\rho$ on $X$ generates a linear subspace $X_{\rho}$ of $X$ naturally defined by

$$
X_{\rho}=\left\{x \in X \mid \lim _{\lambda \rightarrow 0} \rho(\lambda x)=0\right\} .
$$

$X_{\rho}$ is called a modular space.

Definition 2.2. Let $X_{\rho}$ be a modular space and $\left\{x_{n}\right\}$ be a sequence in $X_{\rho}$.

(1) $\left\{x_{n}\right\}$ is $\rho$-convergent to a point $x \in X_{\rho}$ if $\rho\left(x_{n} \rightarrow x_{m}\right) \rightarrow 0$ as $n \rightarrow \infty$. The point $x$ is called a $\rho$-limit of the sequence $\left\{x_{n}\right\}$.

(2) $\left\{x_{n}\right\}$ is called a $\rho$-Cauchy sequence if $\rho\left(x_{n}-x_{m}\right) \rightarrow 0$ as $n, m \rightarrow \infty$.

(3) $X_{\rho}$ is called $\rho$-complete if every $\rho$-Cauchy sequence in $X_{\rho}$ is $\rho$-convergent.

Remark 2.3. If $\rho$ is a convex modular and $0 \leqslant \lambda \leqslant 1$, then we have $\rho(\lambda x) \leqslant \lambda \rho(x)$ for all $x \rightarrow X_{\rho}$. If $\rho$ is a convex modular, and $\lambda_{i} \geqslant 0, i=1,2, \ldots, n$ and $\lambda_{1}+\lambda_{2}+\cdots+\lambda_{n} \leqslant 1$, then $\rho\left(\lambda_{1} x_{1}+\lambda_{2} x_{2}+\cdots+\lambda_{n} x_{n}\right) \leqslant$ $\lambda_{1} \rho\left(x_{1}\right)+\lambda_{2} \rho\left(x_{2}\right)+\cdots+\lambda_{n} \rho\left(x_{n}\right)$. If $\left\{x_{n}\right\}$ is $\rho$-convergent to $x$, then $\left\{\alpha x_{n}\right\}$ is $\rho$-convergent to $\alpha x$, where $0 \leqslant \alpha \leqslant 1$. But the $\rho$-convergence of a sequence $\left\{x_{n}\right\}$ to $x$ does not imply that $\left\{c x_{n}\right\}$ is $\rho$-convergent to $c x$ for scalars $\mathrm{c}$ with $|\mathrm{c}|>1$. 
There are two notions that play important roles when we study modulars. A modular $\rho$ is said to have the Fatou property if $\rho(x) \leqslant \liminf _{n \rightarrow \infty} \rho\left(x_{n}\right)$ for every sequence $\left\{x_{n}\right\}$ that is $\rho$-convergent to $x . \rho$ is said to satisfy the $\Delta_{2}$-condition if there exists a constant $k \geqslant 0$ such that $\rho(2 x) \leqslant k \rho(x)$ for all $x \rightarrow X_{\rho}$.

Example 2.4. For a measure space $(\Omega, \Sigma, \mu)$, let $\mathrm{L}^{0}(\mu)$ be the collection of all measurable functions on $\Omega$. Let

$$
\mathrm{L}^{\phi}(\mu)=\left\{f \rightarrow \mathrm{L}^{0}(\mu) \mid \int_{\Omega} \phi(|\lambda f(x)|) \mathrm{d} \mu(x) \rightarrow 0 \text { as } \lambda \rightarrow 0\right\},
$$

where $\phi:[0, \infty) \rightarrow R$ is assumed to be a continuous, positive, convex and nondecreasing function increasing to infinity with $\phi(0)=0$. We can take, e.g., $\phi(t)=e^{t^{2}}-1$. $L^{\phi}(\mu)$ is called an Orlicz space. Define for $f \rightarrow L^{\phi}(\mu)$,

$$
\rho_{\phi}(f)=\int_{\Omega} \phi(|f|) d \mu .
$$

Then $\rho_{\phi}$ is a complete modular.

\section{Solution of the functional equation (1.3)}

In this section, we solve the mixed type functional equation (1.3). Throughout this section let $\mathrm{E}$ and $\mathrm{H}$ be real vector spaces.

Theorem 3.1. An odd mapping $\mathrm{f}: \mathrm{E} \rightarrow \mathrm{H}$ satisfies the functional equation (1.3) for all $\mathrm{u}, v, w \in \mathrm{E}$ if and only if $f: E \rightarrow H$ satisfies the functional equation (1.1) for all $x, y \in E$.

Proof. Since $f$ is an odd mapping, one can deduce from (1.3) that we have

$$
f\left(\frac{u+v}{2}-w\right)+f\left(\frac{v+w}{2}-u\right)+f\left(\frac{w+u}{2}-v\right)=f(u-v)+f(v-w)+f(w-u)
$$

for all $u, v, w \in E$. Setting $v=u, w=-u$ in (3.1) and finally replacing $u$ by $-x$, we obtain

$$
2 f(x)=f(2 x)
$$

for all $x \in E$. Replacing $x$ by $\frac{x}{2}$ in (3.2), we get

$$
f\left(\frac{x}{2}\right)=\frac{1}{2} f(x)
$$

for all $x \in E$. Letting $u=x, v=y, w=0$ in (3.1) and using the oddness, we obtain

$$
f(x+y)+f(y-2 x)+f(x-2 y)=2 f(x-y)+2 f(y)-2 f(x)
$$

for all $x, y \in E$. Interchanging $x$ and $y$ in (3.3) and using the oddness, we get

$$
f(x+y)+f(x-2 y)+f(-2 x+y)=-2 f(x-y)+2 f(x)-2 f(y)
$$

for all $x, y \in$ E. Subtracting (3.4) from (3.3), we get

$$
f(x-y)=f(x)-f(y)
$$

for all $x, y \in E$. Replacing $y$ by $-y$ and using the oddness, we have (1.1).

Conversly, replacing $(x, y)$ by $(u, u)$ in $(1.1)$, we get $f(2 u)=2 f(u)$ for all $u \in E$.

Replacing $(x, y)$ by $\left(\frac{u+w}{2}, v\right)$ in (1.1) and using the oddness, we get

$$
f\left(\frac{u+w}{2}-v\right)=\frac{1}{2} f(u)+\frac{1}{2} f(w)-f(v)
$$


for all $u, v, w \in E$. Replacing $(x, y)$ by $\left(\frac{v+w}{2}, u\right)$ in (1.1), we get

$$
f\left(\frac{v+w}{2}-u\right)=\frac{1}{2} f(v)+\frac{1}{2} f(w)-f(u)
$$

for all $u, v, w \in E$. Replacing $(x, y)$ by $\left(\frac{u+v}{2}, w\right)$ in (1.1), we get

$$
f\left(\frac{u+v}{2}-w\right)=\frac{1}{2} f(u)+\frac{1}{2} f(v)-f(w)
$$

for all $u, v, w \in E$. Adding (3.5), (3.6), (3.7), and using the oddness, we obtain (3.1) for all $u, v, w \in E$.

Lemma 3.2. Let $\mathrm{f}: \mathrm{E} \rightarrow \mathrm{H}$ satisfy the functional equation (1.2) for all $x, y \in E$. Then $\mathrm{f}: \mathrm{E} \rightarrow \mathrm{H}$ satisfies the functional equation

$$
f(2 x+y)+f(2 x-y)+f(x+2 y)+f(x-2 y)=8 f(x+y)+8 f(x-y)+18 f(x)+18 f(y)
$$

for all $x, y \in E$.

Proof. Let $\mathrm{f}: \mathrm{E} \rightarrow \mathrm{H}$ be a mapping satisfying the functional equation (1.2). Interchanging $\mathrm{x}$ and $\mathrm{y}$ in (1.2) and using eveness of $f$, we arrive at

$$
f(x+2 y)+f(x-2 y)=4(x+y)+4 f(x-y)+24 f(y)-6 f(x) .
$$

Adding (1.2) and (3.9), we get (3.8).

Theorem 3.3. Let an even mapping $\mathrm{f}: \mathrm{E} \rightarrow \mathrm{H}$ satisfies the functional equation (1.3) for all $x, y \in \mathrm{E}$. Then $\mathrm{f}: \mathrm{E} \rightarrow \mathrm{H}$ satisfies the functional equation (3.8) for all $x, y, z \in \mathrm{E}$.

Proof. Since $\mathrm{f}$ is an even mapping, one can deduce from (1.3) that we have

$$
f\left(\frac{u+v}{2}-w\right)+f\left(\frac{v+w}{2}-u\right)+f\left(\frac{w+u}{2}-v\right)=\frac{9}{16}(f(u-v)+f(v-w)+f(w-u))
$$

for all $u, v, w \in E$. Setting $u=v=w=0$ in (3.10), we get $f(0)=0$. Replacing $(u, v, w)$ by $(x, x,-x)$ in (3.10), we get

$$
f(2 x)=16 f(x)
$$

for all $x \in$ E. Setting $x$ by $\frac{x}{2}$ in (3.11), we have

$$
f\left(\frac{x}{2}\right)=\frac{1}{16} f(x)
$$

for all $x \in E$. Replacing $(u, v, w)$ by $(x, y, 0)$ in (3.10) using (3.12) and evenness, we obtain

$$
f(x+y)+f(2 x-y)+f(x-2 y)=9(f(x-y)+f(y)+f(x))
$$

for all $x, y \in E$. Replacing $y$ by $-y$ in (3.13) and using evenness, we obtain

$$
f(x-y)+f(2 x+y)+f(x+2 y)=9(f(x+y)+f(y)+f(x))
$$

for all $x, y \in E$. Adding (3.13) and (3.14), we arrive (3.8) for all $x, y \in E$. By Lemma 3.2, $f$ is quartic. 


\section{Additive-quartic mixed type stability results: direct method}

Throughout this paper, let $V$ and $X$ be linerar spaces, $\rho$ be a convex modular, and $X_{\rho}$ be a $\rho$-complete modular space. Define a mappings $\mathrm{Df}, \mathrm{Df}_{\mathrm{O}}, \mathrm{Df}_{\mathrm{q}_{2}}: \mathrm{V}^{3} \rightarrow \mathrm{X}_{\rho}$ respectively by

$$
\begin{aligned}
D f(u, v, w)=f & \left(\frac{u+v}{2}-w\right)+f\left(\frac{v+w}{2}-u\right)+f\left(\frac{w+u}{2}-v\right) \\
& -\frac{25}{32}(f(u-v)+f(v-w)+f(w-u))+\frac{7}{32}(f(v-u)+f(w-v)+f(u-w))
\end{aligned}
$$

for all $u, v, w \in V$,

$$
D f_{o}(u, v, w)=f\left(\frac{u+v}{2}-w\right)+f\left(\frac{v+w}{2}-u\right)+f\left(\frac{w+u}{2}-v\right)-(f(u-v)+f(v-w)+f(w-u))
$$

for all $u, v, w \in V$, and

$$
D f_{q_{2}}(u, v, w)=f\left(\frac{u+v}{2}-w\right)+f\left(\frac{v+w}{2}-u\right)+f\left(\frac{w+u}{2}-v\right)-\frac{9}{16}(f(u-v)+f(v-w)+f(w-u))
$$

for all $u, v, w \in V$.

\subsection{Additive stability of (1.3) in modular space without $\Delta_{2}$-conditions}

In this subsection, we present the Ulam-Hyers stability of the additive-quartic functional equation (1.3) using Hyers' direct method in modular space without $\Delta_{2}$-conditions.

Theorem 4.1. Let $j \in\{-1,1\}$. Let $\Gamma: V^{3} \rightarrow[0, \infty)$ be a function such that

$$
\sum_{k=0}^{\infty} \frac{\Gamma\left(2^{k j} u, 2^{k j} v, 2^{k j} w\right)}{2^{k j}} \text { converges to } \mathbb{R}
$$

for all $\mathrm{u}, v, w \in \mathrm{V}$ and $\mathrm{f}_{\mathrm{o}}: \mathrm{V} \rightarrow \mathrm{X}_{\rho}$ be an odd mapping that satisfies the inequality

$$
\rho\left(D f_{o}(u, v, w)\right) \leqslant \Gamma(u, v, w)
$$

for all $u, v, w \in V$. Then there exists a unique additive function $\mathrm{A}: \mathrm{V} \rightarrow \mathrm{X}_{\rho}$ such that

$$
\rho\left(f_{o}(u)-A(u)\right) \leqslant \sum_{i=\frac{1-j}{2}}^{\infty} \frac{\Gamma\left(2^{i j} u, 2^{i j} u,-2^{i j} u\right)}{2^{i j}}
$$

for all $\mathrm{u} \in \mathrm{V}$. The mapping $\mathrm{A}(\mathrm{u})$ is defined by

$$
A(u)=\rho-\lim _{k \rightarrow \infty} \frac{f_{o}\left(2^{k j} u\right)}{2^{k j}}
$$

for all $\mathrm{u} \in \mathrm{V}$.

Proof. Assume $\mathfrak{j}=1$. Replacing $(\mathfrak{u}, v, w)$ by $(\mathfrak{u}, \mathfrak{u},-\mathfrak{u})$ in (4.2) and dividing by 2 , we get

$$
\rho\left(\frac{f_{o}(2 u)}{2}-f_{o}(u)\right) \leqslant \frac{1}{2} \Gamma(u, u,-u)
$$

for all $u \in V$, since $f$ is an odd mapping. Replacing $u$ by $2 u$ in (4.5) and dividing by 2 , we get

$$
\rho\left(\frac{f_{o}\left(2^{2} u\right)}{2^{2}}-\frac{f_{o}(2 u)}{2^{2}}\right) \leqslant \frac{1}{2^{2}} \Gamma(2 u, 2 u,-2 u)
$$


for all $u \in V$. Combining (4.5) and (4.6), we obtain

$$
\rho\left(\frac{f_{o}\left(2^{2} u\right)}{2^{4}}-f_{o}(u)\right) \leqslant\left[\frac{1}{2} \Gamma(u, u,-u)+\frac{1}{2^{2}} \Gamma(2 u, 2 u,-2 u)\right]
$$

for all $u \in V$. Using induction on a positive integer $k$, we obtain that

$$
\rho\left(\frac{f_{o}\left(2^{k} u\right)}{2^{k}}-f_{o}(u)\right) \leqslant \sum_{i=0}^{k-1} \frac{1}{2^{i+1}} \Gamma\left(2^{i} u, 2^{i} u,-2^{i} u\right) \leqslant \sum_{i=0}^{\infty} \frac{1}{2^{i+1}} \Gamma\left(2^{i} u, 2^{i} u,-2^{i} u\right)
$$

for all $u \in V$. Let $m$ and $n$ be nonnegative integers with $n>m$. By (4.7), we have

$$
\begin{aligned}
\rho\left(\frac{f_{o}\left(2^{n} u\right)}{2^{n}}-\frac{f_{o}\left(2^{m} u\right)}{2^{m}}\right) & =\rho\left(\frac{1}{2^{m}}\left(\frac{f_{o}\left(2^{n-m} \cdot 2^{m} u\right)}{2^{n-m}}-f_{o}\left(2^{m} u\right)\right)\right) \\
& \leqslant \frac{1}{2^{m}} \sum_{i=0}^{n-m} \frac{1}{2^{i+1}} \Gamma\left(2^{i} \cdot 2^{m} u, 2^{i} \cdot 2^{m} u,-2^{i} \cdot 2^{m} u\right) \\
& =\sum_{i=0}^{n-m} \frac{1}{2^{i+m+1}} \Gamma\left(2^{i+m} u, 2^{i+m} u,-2^{i+m} u\right) \\
& =\sum_{i=m+1}^{n} \frac{1}{2^{i}} \Gamma\left(2^{i-1} u, 2^{i-1} u,-2^{i-1} u\right)
\end{aligned}
$$

for all $u \in V$. Then (4.1) and (4.8) yield that $\left\{\frac{f_{o}\left(2^{k} u\right)}{2^{k}}\right\}$ is a $\rho$-Cauchy sequence in $X_{\rho}$. The $\rho$-completeness of $X_{\rho}$ guarantees its $\rho$-convergence. Hence, there exists a mapping $A: V \rightarrow X_{\rho}$ defined by

$$
A(u)=\rho-\lim _{n \rightarrow \infty} \frac{f_{o}\left(2^{n} u\right)}{2^{n}}, \forall u \in V .
$$

Then we see that

$$
\begin{aligned}
\rho\left(\frac{A(2 u)-2 A(u)}{2^{3}}\right) & =\rho\left(\frac{1}{2^{3}}\left(A(2 u)-\frac{f_{o}\left(2^{n+1} u\right)}{2^{n}}\right)+\frac{1}{2}\left(\frac{1}{2} \cdot \frac{f_{o}\left(2^{n+1}\right)}{2^{n+1}}-\frac{1}{2} A(u)\right)\right) \\
& \leqslant \frac{1}{2^{3}} \rho\left(A(2 u)-\frac{2^{n+1}}{2^{n}}\right)+\frac{1}{4} \rho\left(\frac{f_{o}\left(2^{n+1}\right)}{2^{n+1}}-A(u)\right)
\end{aligned}
$$

for all $u \in V$. Then by (4.9), the right hand side of (4.10) tends to 0 as $n \rightarrow \infty$. Therefore, it follows that

$$
A(2 u)=2 A(u), \quad \forall u \in V .
$$

Next, we calculate $\rho\left(A(u)-f_{o}(u)\right)$. Note that for every $n \in N$, by (4.10) we write

$$
\begin{aligned}
\rho\left(A(u)-f_{o}(u)\right) & =\rho\left(\sum_{k=1}^{n} \frac{f_{o}\left(2^{k} u\right)-2 f_{o}\left(2^{k-1} u\right)}{2^{k}}+\left(A(u)-\frac{f_{o}\left(2^{n} u\right)}{2^{n}}\right)\right) \\
& =\rho\left(\sum_{k=1}^{n} \frac{f_{o}\left(2^{k} u\right)-2 f_{o}\left(2^{k-1} u\right)}{2^{k}}+\frac{1}{2}\left(A(2 u)-\frac{f_{o}\left(2^{n-1} \cdot 2 u\right)}{2^{n-1}}\right)\right) .
\end{aligned}
$$

Since $\sum_{k=1}^{n} \frac{1}{2^{k}}+\frac{1}{2}<1$, it follows from (4.3) and (4.11) that

$$
\begin{aligned}
\rho\left(A(u)-f_{o}(u)\right) & \leqslant \sum_{k=1}^{n} \frac{1}{2^{k}} \rho\left(f_{o}\left(2^{k} u\right)-2 f\left(2^{k-1}\right)\right)+\frac{1}{2} \rho\left(A(2 u)-\frac{f_{o}\left(2^{n-1} \cdot 2 u\right)}{2^{n-1}}\right) \\
& \leqslant \sum_{k=1}^{n} \frac{1}{2^{k}} \Gamma\left(2^{k-1} u, 2^{k-1} u,-2^{k-1} u\right)+\frac{1}{2} \rho\left(A(2 u)-\frac{f_{o}\left(2^{n-1} \cdot 2 u\right)}{2^{n-1}}\right)
\end{aligned}
$$


for all $u \in V$. Letting $n \rightarrow \infty$ in (4.12), we obtain

$$
\rho\left(A(u)-f_{o}(u)\right) \leqslant \Gamma(u, u,-u), \quad \forall u \in V .
$$

Therefore, we get (4.3).

Now, we prove that $A$ is additive. We note that

$$
\rho\left(\frac{1}{2^{n}} D f_{o}\left(2^{n} u, 2^{n} v, 2^{n} w\right)\right) \leqslant \frac{1}{2^{n}} \Gamma\left(2^{n} u, 2^{n} u,-2^{n} u\right) \rightarrow 0 \text { as } n \rightarrow \infty
$$

for all $u, v, w \in \mathrm{V}$. Thus, we observe by convexity of $\rho$ that

$$
\begin{aligned}
\rho( & \frac{1}{7} A\left(\frac{u+v}{2}-w\right)+\frac{1}{7} A\left(\frac{v+w}{2}-u\right)+\frac{1}{7} A\left(\frac{w+u}{2}-v\right) \\
& \left.-\left(\frac{1}{7} A(u-v)+\frac{1}{7} A(v-w)+\frac{1}{7} A(w-u)\right)\right) \\
\leqslant & \frac{1}{7} \rho\left(A\left(\frac{u+v}{2}-w\right)-\frac{1}{2^{n}} f_{o}\left(\frac{2^{n}(u+v)}{2}-2^{n} w\right)\right) \\
& +\frac{1}{7} \rho\left(A\left(\frac{v+w}{2}-u\right)-\frac{1}{2^{n}} f_{o}\left(\frac{2^{n}(v+w)}{2}-2^{n} u\right)\right) \\
& +\frac{1}{7} \rho\left(A\left(\frac{w+u}{2}-v\right)-\frac{1}{2^{n}} f_{o}\left(\frac{2^{n}(w+u)}{2}-2^{n} v\right)\right)+\frac{1}{7} \rho\left(-A(u-v)+\frac{1}{2^{n}} f_{o}\left(2^{n} u-2^{n} v\right)\right) \\
& +\frac{1}{7} \rho\left(-A(v-w)+\frac{1}{2^{n}} f_{o}\left(2^{n} v-2^{n} w\right)\right)+\frac{1}{7} \rho\left(-A(w-u)+\frac{1}{2^{n}} f_{o}\left(2^{n} w-2^{n} u\right)\right) \\
& +\frac{1}{7} \rho\left(\frac { 1 } { 2 ^ { n } } \left(f_{o}\left(\frac{2^{n}(u+v)}{2}-w\right)+f_{o}\left(\frac{2^{n}(v+w)}{2}-u\right)+f_{o}\left(\frac{2^{n}(w+u)}{2}-v\right)\right.\right. \\
& \left.-\left(f_{o}\left(2^{n} u-2^{n} v\right)+f_{o}\left(2^{n} v-2^{n} w\right)+f_{o}\left(2^{n} w-2^{n} u\right)\right)\right)
\end{aligned}
$$

for all $u, v, w \in V$. Taking $n \rightarrow \infty$ in (4.13), we get

$$
A\left(\frac{u+v}{2}-w\right)+A\left(\frac{v+w}{2}-u\right)+A\left(\frac{w+u}{2}-v\right)-(A(u-v)+A(v-w)+A(w-v))=0
$$

for all $u, v, w \in V$. This gives that $A$ is additive.

In order to prove $A$ is unique, let $A^{\prime}$ be another additive mapping satisfying (4.3) and (1.3). Since $A$ and $A^{\prime}$ are additive mappings, $A\left(2^{n} u\right)=2^{n} A(u)$ and $A^{\prime}\left(2^{n} u\right)=2^{n} A^{\prime}(u)$ hold. So

$$
\begin{aligned}
\rho\left(\frac{1}{2} A(u)-\frac{1}{2} A^{\prime}(u)\right) & =\frac{1}{2} \rho\left(\frac{A\left(2^{n} u\right)}{2^{n}}-\frac{f_{o}\left(2^{n} u\right)}{2^{n}}\right)+\frac{1}{2} \rho\left(\frac{f_{o}\left(2^{n} u\right)}{2^{n}}-\frac{A^{\prime}\left(2^{n} u\right)}{2^{n}}\right) \\
& \leqslant \frac{1}{2^{n+1}} \rho\left(A\left(2^{n} u\right)-f_{o}\left(2^{n} u\right)\right)+\frac{1}{2^{n+1}} \rho\left(f_{o}\left(2^{n} u\right)-A^{\prime}\left(2^{n} u\right)\right) \\
& \leqslant \frac{1}{2^{n}} \sum_{i=0}^{\infty} \frac{1}{2^{i+1}} \Gamma\left(2^{i+n} u, 2^{i+n} u,-2^{i+n} u\right) \\
& \leqslant \sum_{i=n}^{\infty} \frac{1}{2^{i+1}} \Gamma\left(2^{i} u, 2^{i} u,-2^{i} u\right) \rightarrow 0 \text { as } n \rightarrow \infty
\end{aligned}
$$

for all $u \in V$. Hence $A$ is unique.

For $j=-1$, we can prove the similar stability result. Hence we complete the proof. 
Corollary 4.2. Let $\Theta$ and $\tau$ be nonnegative real numbers. If an odd mapping $\mathrm{f}_{\mathrm{o}}: \mathrm{V} \rightarrow \mathrm{X}_{\rho}$ satisfies the inequality

$$
\rho\left(D f_{o}(u, v, w)\right) \leqslant \Theta
$$

for all $\mathrm{u}, v, w \in \mathrm{V}$, then there exists a unique additive mapping $\mathrm{A}: \mathrm{V} \rightarrow \mathrm{X}_{\rho}$ such that

$$
\rho\left(f_{o}(u)-A(u)\right) \leqslant \frac{\Theta}{|1|}
$$

for all $u \in V$.

Corollary 4.3. Let $\Theta$ and $\tau$ be nonnegative real numbers with $\tau \neq 1$. If an odd mapping $f_{\mathrm{o}}: \mathrm{V} \rightarrow \mathrm{X}_{\rho}$ satisfies the inequality

$$
\rho\left(\operatorname{Df}_{\mathrm{o}}(u, v, w)\right) \leqslant \Theta\left(\|\mathrm{u}\|^{\tau}+\|v\|^{\tau}+\|w\|^{\tau}\right)
$$

for all $\mathrm{u}, \mathrm{v}, \mathrm{w} \in \mathrm{V}$, then there exists a unique additive mapping $\mathrm{A}: \mathrm{V} \rightarrow \mathrm{X}_{\rho}$ such that

$$
\rho\left(f_{o}(u)-A(u)\right) \leqslant \frac{3 \Theta\|u\|^{\tau}}{\left|2-2^{\tau}\right|}
$$

for all $\mathrm{u} \in \mathrm{V}$.

Corollary 4.4. Let $\Theta$ and $\tau$ be nonnegative real numbers with $\tau \neq \frac{1}{3}$. If an odd mapping $\mathrm{f}_{\mathrm{o}}: \mathrm{V} \rightarrow \mathrm{X}_{\rho}$ satisfies the inequality

$$
\rho\left(\mathrm{Df}_{\mathrm{o}}(u, v, w)\right) \leqslant \Theta\left(\|\mathrm{u}\|^{\tau}\|v\|^{\tau}\|w\|^{\tau}\right)
$$

for all $\mathrm{u}, \mathrm{v}, \mathrm{w} \in \mathrm{V}$, then there exists a unique additive mapping $\mathrm{A}: \mathrm{V} \rightarrow \mathrm{X}_{\rho}$ such that

$$
\rho\left(f_{o}(u)-A(u)\right) \leqslant \frac{\Theta\|u\|^{3 \tau}}{\left|2-2^{3 \tau}\right|}
$$

for all $\mathrm{u} \in \mathrm{V}$.

Corollary 4.5. Let $\Theta$ and $\tau$ be nonnegative real numbers with $\tau \neq \frac{1}{3}$. If an odd mapping $\mathrm{f}_{\mathrm{o}}: \mathrm{V} \rightarrow \mathrm{X}_{\rho}$ satisfies the inequality

$$
\rho\left(\operatorname{Df}_{\mathrm{o}}(\mathrm{u}, v, w)\right) \leqslant \Theta\left(\|\mathrm{u}\|^{\tau}\|v\|^{\tau}\|w\|^{\tau}+\|u\|^{3 \tau}+\|v\|^{3 \tau}+\|w\|^{3 \tau}\right)
$$

for all $\mathrm{u}, \mathrm{v}, \mathrm{w} \in \mathrm{V}$, then there exists a unique additive mapping $\mathrm{A}: \mathrm{V} \rightarrow \mathrm{X}_{\rho}$ such that

$$
\rho\left(f_{o}(u)-A(u)\right) \leqslant \frac{4 \Theta \|\left. u\right|^{3 \tau}}{\left|2-2^{3 \tau}\right|}
$$

for all $\mathrm{u} \in \mathrm{V}$.

\subsection{Additive stability of (1.3) in modular space with $\Delta_{2}$-conditions}

In this subsection, we present the Ulam-Hyers stability of the additive-quartic functional equation (1.3) using Hyers' direct method in modular space with $\Delta_{2}$-conditions.

Theorem 4.6. Let $\mathrm{j} \in\{-1,1\}$. Let $\Gamma: \mathrm{V}^{3} \rightarrow[0, \infty)$ be a function such that

$$
\sum_{n=0}^{\infty}\left(\frac{k^{2}}{2}\right)^{n} \Gamma\left(\frac{u}{2^{n j}}, \frac{u}{2^{n j}}, \frac{-u}{2^{n j}}\right)<+\infty \quad \text { and } \quad \lim _{n \rightarrow \infty} k^{n j} \Gamma\left(\frac{u}{2^{n j}}, \frac{v}{2^{n j}}, \frac{w}{2^{n j}}\right)=0
$$


for all $\mathrm{u}, \mathrm{v}, \mathrm{w} \in \mathrm{V}$ and $\mathrm{f}_{\mathrm{o}}: \mathrm{V} \rightarrow \mathrm{X}_{\rho}$ be an odd mapping satisfying the inequality

$$
\rho\left(D f_{o}(u, v, w)\right) \leqslant \Gamma(u, v, w)
$$

for all $\mathrm{u}, v, w \in \mathrm{V}$. Then there exists a unique additive mapping $\mathrm{A}: \mathrm{X} \rightarrow \mathrm{H}$ such that

$$
\rho\left(f_{o}(u)-A(u)\right) \leqslant \frac{1}{2} \sum_{i=\frac{1-j}{2}}^{\infty}\left(\frac{k^{2}}{2}\right)^{i} \Gamma\left(\frac{u}{2^{i j}}, \frac{u}{2^{i j}}, \frac{-u}{2^{i j}}\right)
$$

for all $\mathrm{u} \in \mathrm{V}$. The mapping $\mathrm{A}(\mathrm{u})$ is defined by

$$
A(u)=\rho-\lim _{n \rightarrow \infty} 2^{n j} f_{o}\left(\frac{u}{2^{n j}}\right)
$$

for all $\mathrm{u} \in \mathrm{V}$.

Proof. Assume $j=1$. Replacing $(u, v, w)$ by $\left(\frac{u}{2}, \frac{u}{2}, \frac{-u}{2}\right)$ in (4.15), we get

$$
\rho\left(f_{o}(u)-2 f_{o}\left(\frac{u}{2}\right)\right) \leqslant \Gamma\left(\frac{u}{2}, \frac{u}{2}, \frac{-u}{2}\right)
$$

for all $u \in V$, since $f$ is an odd mapping. Then it follows from $\Delta_{2}$-condition and the convexity of the modular $\rho$ that

$$
\rho\left(f_{o}(u)-2^{n} f_{o}\left(\frac{u}{2^{n}}\right)\right)=\rho\left(\sum_{i=1}^{n} \frac{1}{2^{i}}\left(f_{o}\left(\frac{u}{2^{i-1}}\right)-2^{2 i} f_{o}\left(\frac{u}{2^{i}}\right)\right)\right) \leqslant \frac{1}{k} \sum_{i=1}^{n}\left(\frac{k^{2}}{2}\right)^{i} \Gamma\left(\frac{u}{2^{i}}, \frac{u}{2^{i}}, \frac{-u}{2^{i}}\right)
$$

for all $u \in V$. So, for all $n, m \in \mathbb{N}$ with $n \geqslant m$, we have

$$
\begin{aligned}
\rho\left(2^{n} f_{o}\left(\frac{u}{2^{n}}\right)-2^{m} f_{o}\left(\frac{u}{2^{m}}\right)\right) & =\rho\left(2^{m}\left(2^{n-m} f_{o}\left(\frac{u}{2^{n-m}}\right)-f_{o}\left(\frac{u}{2^{m}}\right)\right)\right) \\
& \leqslant k^{m} \sum_{i=1}^{n-m}\left(\frac{k^{2}}{2}\right)^{i} \Gamma\left(\frac{u}{2^{i} \cdot 2^{m}}, \frac{u}{2^{i} \cdot 2^{m}}, \frac{-u}{2^{i} \cdot 2^{m}}\right) \\
& \leqslant \frac{2^{m}}{k^{m+1}} \sum_{i=m+1}^{n}\left(\frac{k^{2}}{2}\right)^{2} \Gamma\left(\frac{u}{2^{i}}, \frac{u}{2^{i}}, \frac{-u}{2^{i}}\right)
\end{aligned}
$$

for all $u \in V$. Since the right hand side of the above inequality tends to zero as $n$ goes to infinity, the sequence $\left\{2^{n} f_{o}\left(\frac{u}{2^{n}}\right)\right\}$ is a $\rho$-Cauchy sequence in $X_{\rho}$ and so the sequence $\left\{2^{n} f_{o}\left(\frac{u}{2^{n}}\right)\right\}$ is a $\rho$-convergent sequence on $X_{\rho}$. Thus, we may define a mapping $A: V \rightarrow X_{\rho}$ as

$$
A(u)=\rho-\lim _{n \rightarrow \infty} 2^{n} f_{o}\left(\frac{u}{2^{n}}\right) \text {, i.e., } \lim _{n \rightarrow \infty} \rho\left(2^{n} f_{o}\left(\frac{u}{2^{n}}\right)-A(u)\right)=0
$$

for all $u \in V$. According to the $\Delta_{2}$-condition, we obtain the following inequality

$$
\begin{aligned}
\rho(A(u)-f(u)) & \leqslant \frac{1}{2} \rho\left(2 A(u)-2^{n+1} f_{o}\left(\frac{u}{2^{n}}\right)\right)+\frac{1}{2} \rho\left(2^{n+1} f_{o}\left(\frac{u}{2^{n}}\right)-2 f(u)\right) \\
& \leqslant \frac{k}{2} \rho\left(A(u)-2^{n} f_{o}\left(\frac{u}{2^{n}}\right)\right)+\frac{k}{2} \rho\left(2^{n} f_{o}\left(\frac{u}{2^{n}}\right)-f(u)\right) \\
& \leqslant \frac{1}{2} \sum_{i=1}^{\infty}\left(\frac{k^{2}}{2}\right)^{i} \Gamma\left(\frac{u}{2^{i}}, \frac{u}{2^{i}}, \frac{-u}{2^{i}}\right)+\frac{k}{2} \rho\left(2^{n} f_{o}\left(\frac{u}{2^{n}}\right)-f(u)\right)
\end{aligned}
$$


for all $u \in V$. Taking $n$ tends to $\infty$, we conclude that the estimation (4.16) of $f$ by $A$ holds for all $u \in V$. Now, we claim that the mapping $A$ is additive. Replacing $(u, v, w)$ by $\left(\frac{u}{2^{n}}, \frac{v}{2^{n}}, \frac{w}{2^{n}}\right)$ in $(4.15)$ with $\Delta_{2}$-condition, we obtain

$$
\rho\left(2^{n} \operatorname{Df}_{o}\left(\frac{u}{2^{n}}, \frac{v}{2^{n}}, \frac{w}{2^{n}}\right)\right) \leqslant k^{n} \Gamma\left(\frac{u}{2^{n}}, \frac{v}{2^{n}}, \frac{w}{2^{n}}\right)
$$

for all $u, v, w \in V$. Thus, it follows from the $\Delta_{2}$-condition such that

$$
\begin{aligned}
\rho(A & \left.\left(\frac{u+v}{2}-w\right)+A\left(\frac{v+w}{2}-u\right)+A\left(\frac{w+u}{2}-v\right)-(A(u-v)+A(v-w)+A(w-u))\right) \\
\leqslant & \frac{1}{7} \rho\left(7\left(A\left(\frac{u+v}{2}-w\right)-2^{n} f_{o}\left(\frac{u+v}{2^{n+1}}-\frac{w}{2^{n}}\right)\right)\right) \\
& +\frac{1}{7} \rho\left(7\left(A\left(\frac{v+w}{2}-u\right)-2^{n} f_{o}\left(\frac{v+w}{2^{n+1}}-\frac{u}{2^{n}}\right)\right)\right) \\
& +\frac{1}{7} \rho\left(7\left(A\left(\frac{w+u}{2}-v\right)-2^{n} f_{o}\left(\frac{w+u}{2^{n+1}}-\frac{v}{2^{n}}\right)\right)\right)+\frac{1}{7} \rho\left(7\left(-A(u-v)+2^{n} f_{o}\left(\frac{u-v}{2^{n}}\right)\right)\right) \\
+ & \frac{1}{7} \rho\left(7\left(-A(v-w)+2^{n} f_{o}\left(\frac{v-w}{2^{n}}\right)\right)\right)+\frac{1}{7} \rho\left(7\left(-A(w-u)+2^{n} f_{o}\left(\frac{w-u}{2^{n}}\right)\right)\right) \\
+ & \frac{1}{7} \rho\left(7 \left(2 ^ { n } \left(f_{o}\left(\frac{u+v}{2^{n+1}}-\frac{w}{2^{n}}\right)+f_{o}\left(\frac{v+w}{2^{n+1}}-\frac{u}{2^{n}}\right)+f_{o}\left(\frac{w+u}{2^{n+1}}-\frac{v}{2^{n}}\right)\right.\right.\right. \\
& \left.\left.\left.-\left(f_{o}\left(\frac{u-v}{2^{n}}\right)+f_{o}\left(\frac{v-w}{2^{n}}\right)+f_{o}\left(\frac{w-u}{2^{n}}\right)\right)\right)\right)\right) \\
\leqslant & \frac{k}{7} \rho\left(A\left(\frac{u+v}{2}-w\right)-2^{n} f_{o}\left(\frac{u+v}{2^{n+1}}-\frac{w}{2^{n}}\right)\right)+\frac{k}{7} \rho\left(A\left(\frac{v+w}{2}-u\right)-2^{n} f_{o}\left(\frac{v+w}{2^{n+1}}-\frac{u}{2^{n}}\right)\right) \\
+ & \frac{k}{7} \rho\left(A\left(\frac{w+u}{2}-v\right)-2^{n} f_{o}\left(\frac{w+u}{2^{n+1}}-\frac{v}{2^{n}}\right)\right)+\frac{k}{7} \rho\left(-A(u-v)+2^{n} f_{o}\left(f_{o}\left(\frac{u-v}{2^{n}}\right)\right)\right) \\
+ & \frac{k}{7} \rho\left(-A(v-w)+2^{n} f_{o}\left(f_{o}\left(\frac{v-w}{2^{n}}\right)\right)\right)+\frac{k}{7} \rho\left(-A(w-u)+2^{n} f_{o}\left(f_{o}\left(\frac{w-u}{2^{n}}\right)\right)\right) \\
+ & \frac{k}{7} \rho\left(2 ^ { n } \left(f_{o}\left(\frac{u+v}{2^{n+1}}-\frac{w}{2^{n}}\right)+f_{o}\left(\frac{v+w}{2^{n+1}}-\frac{u}{2^{n}}\right)+f_{o}\left(\frac{w+u}{2^{n+1}}-\frac{v}{2^{n}}\right)\right.\right. \\
& \left.\left.-\left(f_{o}\left(\frac{u-v}{2^{n}}\right)+f_{o}\left(\frac{v-w}{2^{n}}\right)+f_{o}\left(\frac{w-u}{2^{n}}\right)\right)\right)\right)
\end{aligned}
$$

for all $u, v, w \in V$ and all positive integers $n$. Let us take the limit as $n$ tends to $\infty$, one see that $A$ is additive. In order to prove $A$ is unique, let $A^{\prime}(u)$ be another additive mapping satisfying (4.16) and (1.3). Since $A$ and $A^{\prime}$ are additive mappings, $A\left(\frac{u}{2^{n}}\right)=\frac{1}{2^{n}} A(u)$ and $A^{\prime}\left(\frac{u}{2^{n}}\right)=\frac{1}{2^{n}} A^{\prime}(u)$ hold. Thus

$$
\begin{aligned}
\rho\left(A(u)-A^{\prime}(u)\right) & =\frac{1}{2} \rho\left(2^{n+1} A\left(\frac{u}{2^{n}}\right)-2^{n+1} f_{o}\left(\frac{u}{2^{n}}\right)\right)+\frac{1}{2} \rho\left(2^{n+1} f_{o}\left(\frac{u}{2^{n}}\right)-2^{n+1} A^{\prime}\left(\frac{u}{2^{n}}\right)\right) \\
& \leqslant \frac{k^{n+1}}{2} \rho\left(A\left(\frac{u}{2^{n}}\right)-f_{o}\left(\frac{u}{2^{n}}\right)\right)+\frac{k^{n+1}}{2} \rho\left(f_{o}\left(\frac{u}{2^{n}}\right)-A^{\prime}\left(\frac{u}{2^{n}}\right)\right) \\
& \leqslant\left(\frac{2}{k}\right)^{n-1} \sum_{i=n+1}^{\infty}\left(\frac{k^{2}}{2}\right)^{i} \Gamma\left(\frac{u}{2^{i}}, \frac{u}{2^{i}}, \frac{-u}{2^{i}}\right) \rightarrow 0 \text { as } n \rightarrow \infty
\end{aligned}
$$

for all $u \in V$. Hence $A$ is unique.

For $j=-1$, we can prove the similar stability result. Hence we complete the proof.

Corollary 4.7. Let $\Theta$ and $\tau$ be nonnegative real numbers and $X_{\rho}$ satisfies $\Delta_{2}$ condition. If an odd mapping $\mathrm{f}_{\mathrm{o}}: \mathrm{V} \rightarrow \mathrm{X}_{\rho}$ satisfies the inequality

$$
\rho\left(D f_{o}(u, v, w)\right) \leqslant \Theta
$$


for all $\mathrm{u}, v, w \in \mathrm{V}$, hen there exists a unique additive mapping $\mathrm{A}: \mathrm{V} \rightarrow \mathrm{X}_{\rho}$ such that

$$
\rho\left(f_{o}(u)-A(u)\right) \leqslant \frac{k^{2} \Theta}{|1|}
$$

for all $u \in V$.

Corollary 4.8. Let $\Theta$ and $\tau$ be nonnegative real numbers and $X_{\rho}$ satisfies $\Delta_{2}$ condition with $2^{\tau} \neq k^{2}$. If an odd mapping $\mathrm{f}_{\mathrm{o}}: \mathrm{V} \rightarrow \mathrm{X}_{\rho}$ satisfies the inequality

$$
\rho\left(D f_{o}(u, v, w)\right) \leqslant \Theta\left(\|u\|^{\tau}+\|v\|^{\tau}+\|w\|^{\tau}\right)
$$

for all $\mathrm{u}, v, w \in \mathrm{V}$, then there exists a unique additive mapping $\mathrm{A}: \mathrm{V} \rightarrow \mathrm{X}_{\rho}$ such that

$$
\rho\left(f_{o}(u)-A(u)\right) \leqslant \frac{3 k^{2} \Theta\|u\|}{\left|k^{2}-2^{\tau}\right|}
$$

for all $\mathrm{u} \in \mathrm{V}$.

Corollary 4.9. Let $\Theta$ and $\tau$ be nonnegative real numbers and $X_{\rho}$ satisfies $\Delta_{2}$ condition with $2^{3 \tau} \neq k^{2}$. If an odd mapping $\mathrm{f}_{\mathrm{o}}: \mathrm{V} \rightarrow \mathrm{X}_{\rho}$ satisfies the inequality

$$
\rho\left(\operatorname{Df}_{\mathrm{o}}(u, v, w)\right) \leqslant \Theta\left(\|u\|^{\tau}\|v\|^{\tau}\|w\|^{\tau}\right)
$$

for all $\mathrm{u}, v, w \in \mathrm{V}$, then there exists a unique additive mapping $\mathrm{A}: \mathrm{V} \rightarrow \mathrm{X}_{\rho}$ such that

$$
\rho\left(f_{o}(u)-A(u)\right) \leqslant \frac{k^{2} \Theta \| u||^{3 \tau}}{\left|k^{2}-2^{3 \tau}\right|}
$$

for all $\mathrm{u} \in \mathrm{V}$.

Corollary 4.10. Let $\Theta$ and $\tau$ be nonnegative real numbers and $X_{\rho}$ satisfies $\Delta_{2}$ condition with $2^{3 \tau} \neq k^{2}$. If an odd mapping $\mathrm{f}_{\mathrm{o}}: \mathrm{V} \rightarrow \mathrm{X}_{\rho}$ satisfies the inequality

$$
\rho\left(D f_{o}(u, v, w)\right) \leqslant \Theta\left(\|u\|^{\tau}\|v\|^{\tau}\|w\|^{\tau}+\|u\|^{3 \tau}+\|v\|^{3 \tau}+\|w\|^{3 \tau}\right)
$$

for all $\mathrm{u}, v, w \in \mathrm{V}$, then there exists a unique additive mapping $\mathrm{A}: \mathrm{V} \rightarrow \mathrm{X}_{\rho}$ such that

$$
\rho\left(f_{o}(u)-A(u)\right) \leqslant \frac{4 k^{2} \Theta|| u||^{3 \tau}}{\left|k^{2}-2^{3 \tau}\right|}
$$

for all $\mathrm{u} \in \mathrm{V}$.

4.3. Quartic stability of (1.3) in modular space without $\Delta_{2}$-conditions

In this subsection, we present the Ulam-Hyers stability of the additive-quartic functional equation (1.3) using Hyers' direct method in modular space without $\Delta_{2}$-conditions.

Theorem 4.11. Let $j \in\{-1,1\}$. Let $\Gamma: \mathrm{V}^{3} \rightarrow[0, \infty)$ be a function such that

$$
\sum_{k=0}^{\infty} \frac{\Gamma\left(2^{k j} u, 2^{k j} v, 2^{k j} w\right)}{2^{4 k j}}<\infty
$$

for all $\mathrm{u}, v, w \in \mathrm{V}$ and $\mathrm{f}_{\mathrm{q}_{2}}: \mathrm{V} \rightarrow \mathrm{X}_{\rho}$ be an even mapping satisfying the inequality

$$
\rho\left(D f_{q_{2}}(u, v, w)\right) \leqslant \Gamma(u, v, w)
$$


for all $\mathrm{u}, v, w \in \mathrm{V}$. Then there exists a unique quartic mapping $\mathrm{Q}_{2}: \mathrm{V} \rightarrow \mathrm{X}_{\rho}$ such that

$$
\rho\left(f_{q_{2}}(u)-Q_{2}(u)\right) \leqslant \sum_{i=\frac{1-j}{2}}^{\infty} \frac{\Gamma\left(2^{i j} u, 2^{i j} u,-2^{i j} u\right)}{2^{4 i j}}
$$

for all $\mathrm{u} \in \mathrm{V}$. The mapping $\mathrm{Q}_{2}(\mathrm{u})$ is defined by

$$
\mathrm{Q}_{2}(\mathrm{u})=\rho-\lim _{k \rightarrow \infty} \frac{\mathrm{f}_{\mathrm{q}_{2}}\left(2^{\mathrm{kj}} \mathrm{u}\right)}{2^{4 k j}}
$$

for all $\mathrm{u} \in \mathrm{V}$.

Proof. Replacing $(u, v, w)$ by $(u, u,-u)$ and dividing by $2^{4}$, we get

$$
\rho\left(\frac{f_{\mathrm{q}_{2}}(2 u)}{2^{4}}-f_{\mathrm{q}_{2}}(\mathrm{u})\right) \leqslant \frac{1}{2^{4}} \Gamma(\mathrm{u}, \mathrm{u},-\mathrm{u})
$$

for all $u \in V$, since $f$ is an even mapping. Then the conclusion is a direct consequence of Theorem 4.1.

Corollary 4.12. Let $\Theta$ and $\tau$ be nonnegative real numbers. If an even mapping $\mathrm{f}_{\mathrm{q}_{2}}: \mathrm{V} \rightarrow \mathrm{X}_{\rho}$ satisfies the inequality

$$
\rho\left(\mathrm{Df}_{\mathrm{q}_{2}}(\mathrm{u}, v, w)\right) \leqslant \Theta
$$

for all $\mathrm{u}, v, w \in \mathrm{V}$, then there exists a unique quartic mapping $\mathrm{Q}_{2}: \mathrm{V} \rightarrow \mathrm{X}_{\rho}$ such that

$$
\rho\left(f_{q_{2}}(u)-Q_{2}(u)\right) \leqslant \frac{\Theta}{|15|}
$$

for all $\mathrm{u} \in \mathrm{V}$.

Corollary 4.13. Let $\Theta$ and $\tau$ be nonnegative real numbers with $\tau \neq 4$. If an even mapping $\mathrm{f}_{\mathrm{q}_{2}}: \mathrm{V} \rightarrow \mathrm{X}_{\rho}$ satisfies the inequality

$$
\rho\left(D f_{q_{2}}(u, v, w)\right) \leqslant \Theta\left(\|u\|^{\tau}+\|v\|^{\tau}+\|w\|^{\tau}\right)
$$

for all $\mathrm{u}, v, w \in \mathrm{V}$, then there exists a unique quartic mapping $\mathrm{Q}_{2}: \mathrm{V} \rightarrow \mathrm{X}_{\rho}$ such that

$$
\rho\left(f_{q_{2}}(u)-Q_{2}(u)\right) \leqslant \frac{3 \Theta\|u\| \tau}{\left|2^{4}-2^{\tau}\right|}
$$

for all $u \in V$.

Corollary 4.14. Let $\Theta$ and $\tau$ be nonnegative real numbers with $\tau \neq \frac{4}{3}$. If an odd mapping $\mathrm{f}_{\mathrm{q}_{2}}: \mathrm{V} \rightarrow \mathrm{X}_{\rho}$ satisfies the inequality

$$
\rho\left(\mathrm{Df}_{\mathrm{q}_{2}}(\mathrm{u}, v, w)\right) \leqslant \Theta\left(\|u\|^{\tau}\|v\|^{\tau}\|w\|^{\tau}\right)
$$

for all $\mathrm{u}, v, w \in \mathrm{V}$, then there exists a unique quartic mapping $\mathrm{Q}_{2}: \mathrm{V} \rightarrow \mathrm{X}_{\rho}$ such that

$$
\rho\left(f_{q_{2}}(u)-Q_{2}(u)\right) \leqslant \frac{\Theta\|u\|^{3 \tau}}{\left|2^{4}-2^{3 \tau}\right|}
$$

for all $\mathrm{u} \in \mathrm{V}$.

Corollary 4.15. Let $\Theta$ and $\tau$ be nonnegative real numbers with $\tau \neq \frac{4}{3}$. If an even mapping $\mathrm{f}_{\mathrm{q}_{2}}: \mathrm{V} \rightarrow \mathrm{X}_{\rho}$ satisfies the inequality

$$
\rho\left(\mathrm{Df}_{\mathrm{q}_{2}}(u, v, w)\right) \leqslant \Theta\left(\|u\|^{\tau}\|v\|^{\tau}\|w\|^{\tau}+\|u\|^{3 \tau}+\|v\|^{3 \tau}+\|w\|^{3 \tau}\right)
$$

for all $\mathrm{u}, v, w \in \mathrm{V}$, then there exists a unique quartic mapping $\mathrm{Q}_{2}: \mathrm{V} \rightarrow \mathrm{X}_{\rho}$ such that

$$
\rho\left(f_{q_{2}}(u)-Q_{2}(u)\right) \leqslant \frac{4 \Theta \|\left. u\right|^{3 \tau}}{\left|2^{4}-2^{3 \tau}\right|}
$$

for all $\mathrm{u} \in \mathrm{V}$. 
4.4. Quartic stability of (1.3) in modular space with $\Delta_{2}$-conditions

In this subsection, we present the Ulam-Hyers stability of the additive-quartic functional equation (1.3) using Hyers' direct method in modular space with $\Delta_{2}$-conditions.

Theorem 4.16. Let $j \in\{-1,1\}$. Let $\Gamma: \mathrm{V}^{3} \rightarrow[0, \infty)$ be a function such that

$$
\sum_{n=0}^{\infty}\left(\frac{k^{8}}{2^{4}}\right)^{n} \Gamma\left(\frac{u}{2^{n j}}, \frac{u}{2^{n j}}, \frac{-u}{2^{n j}}\right)<\infty \text { and } \lim _{n \rightarrow \infty} k^{4 n j} \Gamma\left(\frac{u}{2^{n j}}, \frac{v}{2^{n j}}, \frac{w}{2^{n j}}\right)=0
$$

for all $\mathrm{u}, v, w \in \mathrm{V}$ and $\mathrm{f}_{\mathrm{q}_{2}}: \mathrm{V} \rightarrow \mathrm{X}_{\rho}$ be an even mapping satisfying the inequality

$$
\rho\left(D f_{q_{2}}(u, v, w)\right) \leqslant \Gamma(u, v, w)
$$

for all $\mathrm{u}, v, w \in \mathrm{V}$. Then there exists a unique quartic mapping $\mathrm{Q}_{2}: \mathrm{V} \rightarrow \mathrm{X}_{\rho}$ such that

$$
\rho\left(f_{q_{2}}(u)-Q_{2}(u)\right) \leqslant \frac{1}{2^{4}} \sum_{i=\frac{1-j}{2}}^{\infty}\left(\frac{k^{8}}{16}\right)^{i} \Gamma\left(\frac{u}{2^{i j}}, \frac{u}{2^{i j}}, \frac{-u}{2^{i j}}\right)
$$

for all $\mathrm{u} \in \mathrm{V}$. The mapping $\mathrm{Q}_{2}(\mathrm{u})$ is defined by

$$
\mathrm{Q}_{2}(\mathrm{u})=\rho-\lim _{\mathrm{n} \rightarrow \infty} 2^{4 n j} \mathrm{f}_{\mathrm{q}_{2}}\left(\frac{\mathrm{u}}{2^{\mathrm{nj}}}\right)
$$

for all $\mathrm{u} \in \mathrm{V}$.

Proof. Assume $j=1$. Replacing $(u, v, w)$ by $\left(\frac{u}{2}, \frac{u}{2}, \frac{-u}{2}\right)$ in $(4.21)$, we get

$$
\rho\left(\mathrm{f}_{\mathrm{q}_{2}}(\mathrm{u})-2^{4} \mathrm{f}_{\mathrm{q}_{2}}\left(\frac{\mathrm{u}}{2}\right)\right) \leqslant \Gamma\left(\frac{\mathrm{u}}{2}, \frac{\mathrm{u}}{2}, \frac{-\mathrm{u}}{2}\right)
$$

for all $u \in V$, since $f$ is an even mapping. Then the conclusion is a direct consequence of Theorem 4.6.

Corollary 4.17. Let $\Theta$ and $\tau$ be nonnegative real numbers and $X_{\rho}$ satisfies $\Delta_{2}$ condition. If an even mapping $\mathrm{f}_{\mathrm{q}_{2}}: \mathrm{V} \rightarrow \mathrm{X}_{\rho}$ satisfies the inequality

$$
\rho\left(\mathrm{Df}_{\mathrm{q}_{2}}(\mathrm{u}, v, w)\right) \leqslant \Theta
$$

for all $\mathrm{u}, \mathrm{v}, \mathrm{w} \in \mathrm{V}$, then there exists a unique quartic mapping $\mathrm{Q}_{2}: \mathrm{V} \rightarrow \mathrm{X}_{\rho}$ such that

$$
\rho\left(f_{q_{2}}(u)-Q_{2}(u)\right) \leqslant \frac{k^{8} \Theta}{|15|}
$$

for all $u \in V$.

Corollary 4.18. Let $\Theta$ and $\tau$ be nonnegative real numbers and $X_{\rho}$ satisfies $\Delta_{2}$ condition with $2^{\tau} \neq \mathrm{k}^{8}$. If an even mapping $\mathrm{f}_{\mathrm{q}_{2}}: \mathrm{V} \rightarrow \mathrm{X}_{\rho}$ satisfies the inequality

$$
\rho\left(\operatorname{Df}_{q_{2}}(u, v, w)\right) \leqslant \Theta\left(\|u\|^{\tau}+\|v\|^{\tau}+\|w\|^{\tau}\right)
$$

for all $\mathrm{u}, \mathrm{v}, \mathrm{w} \in \mathrm{V}$, then there exists a unique quartic mapping $\mathrm{Q}_{2}: \mathrm{V} \rightarrow \mathrm{X}_{\rho}$ such that

$$
\rho\left(f_{q_{2}}(u)-Q_{2}(u)\right) \leqslant \frac{3 k^{8} \Theta \|\left. u\right|^{\tau}}{\left|k^{8}-2^{\tau}\right|}
$$

for all $u \in V$. 
Corollary 4.19. Let $\Theta$ and $\tau$ be nonnegative real numbers and $X_{\rho}$ satisfies $\Delta_{2}$ condition with $2^{3 \tau} \neq k^{8}$. If an even mapping $\mathrm{f}_{\mathrm{q}_{2}}: \mathrm{V} \rightarrow \mathrm{X}_{\rho}$ satisfies the inequality

$$
\rho\left(\mathrm{Df}_{\mathrm{q}_{2}}(\mathrm{u}, v, w)\right) \leqslant \Theta\left(\|u\|^{\tau}\|v\|^{\tau}\|w\|^{\tau}\right)
$$

for all $\mathrm{u}, v, w \in \mathrm{V}$, then there exists a unique quartic mapping $\mathrm{Q}_{2}: \mathrm{V} \rightarrow \mathrm{X}_{\rho}$ such that

$$
\rho\left(f_{q_{2}}(u)-Q_{2}(u)\right) \leqslant \frac{k^{8} \Theta \|\left. u\right|^{3 \tau}}{\left|k^{8}-2^{3 \tau}\right|}
$$

for all $\mathrm{u} \in \mathrm{V}$.

Corollary 4.20. Let $\Theta$ and $\tau$ be nonnegative real numbers and $X_{\rho}$ satisfies $\Delta_{2}$ condition with $2^{3 \tau} \neq k^{8}$. If an even mapping $\mathrm{f}_{\mathrm{q}_{2}}: \mathrm{V} \rightarrow \mathrm{X}_{\rho}$ satisfies the inequality

$$
\rho\left(\operatorname{Df}_{\mathrm{q}_{2}}(u, v, w)\right) \leqslant \Theta\left(\|u\|^{\tau}\|v\|^{\tau}\|w\|^{\tau}+\|u\|^{3 \tau}+\|v\|^{3 \tau}+\|w\|^{3 \tau}\right)
$$

for all $\mathrm{u}, \mathrm{v}, \mathrm{w} \in \mathrm{V}$, then there exists a unique quartic mapping $\mathrm{Q}_{2}: \mathrm{V} \rightarrow \mathrm{X}_{\rho}$ such that

$$
\rho\left(f_{q_{2}}(u)-Q_{2}(u)\right) \leqslant \frac{4 k^{8} \Theta \| u||^{3 \tau}}{\left|k^{8}-2^{3 \tau}\right|}
$$

for all $\mathrm{u} \in \mathrm{V}$.

4.5. Additive-quartic stability of (1.3) in modular space without $\Delta_{2}$-conditions

In this subsection, we present the Ulam -Hyers stability of the additive-quartic mixed type functional equation (1.3) using Hyers' direct method in modular space without $\Delta_{2}$-conditions.

Theorem 4.21. Let $\mathrm{j} \in\{-1,1\}$. Let $\Gamma: \mathrm{V} \rightarrow[0, \infty)$ be a function satisfying (4.1) and (4.18) for all $u, v, w \in \mathrm{V}$. Let $\mathrm{f}: \mathrm{V} \rightarrow \mathrm{X}_{\rho}$ be a mapping satisfying the inequality

$$
\rho(\operatorname{Df}(u, v, w)) \leqslant \Gamma(u, v, w)
$$

for all $\mathrm{u}, v, w \in \mathrm{V}$. Then there exists a unique additive mapping $\mathrm{A}: \mathrm{V} \rightarrow \mathrm{X}_{\rho}$ and a unique quartic mapping $\mathrm{Q}_{2}: \mathrm{V} \rightarrow \mathrm{X}_{\rho}$ such that

$$
\rho\left(f(u)-A(u)-Q_{2}(u)\right) \leqslant \frac{1}{2}\left\{\sum_{i=\frac{1-j}{2}}^{\infty} \frac{\Gamma\left(2^{i j} u, 2^{i j} u,-2^{i j} u\right)}{2^{i j}}+\sum_{i=\frac{1-j}{2}}^{\infty} \frac{\Gamma\left(2^{i j} u, 2^{i j} u,-2^{i j} u\right)}{2^{4 i j}}\right\}
$$

for all $\mathrm{u} \in \mathrm{V}$. The mappings $\mathrm{A}(\mathrm{u})$ and $\mathrm{Q}_{2}(\mathrm{u})$ respectively are defined in (4.4) and (4.19).

Proof. Let $\mathrm{f}_{\mathrm{q}_{2}}(\mathrm{u})=\frac{1}{2}\{f(u)+f(-u)\}$ for all $u \in V$. Then $\mathrm{f}_{\mathrm{q}_{2}}(0)=0, \mathrm{f}_{\mathrm{q}_{2}}(\mathrm{u})=\mathrm{f}_{\mathrm{q}_{2}}(-\mathrm{u})$. Hence

$$
\begin{aligned}
\rho\left(\operatorname{Df}_{\mathrm{q}_{2}}(\mathrm{u}, v, w)\right) & =\frac{1}{2}\{\rho(\operatorname{Df}(u, v, w)+\operatorname{Df}(-\mathfrak{u},-v,-w))\} \\
& \leqslant \frac{1}{2}\{\rho(\operatorname{Df}(u, v, w))+\rho(\operatorname{Df}(-u,-v,-w))\} \leqslant \frac{1}{2}\{\Gamma(u, v, w)+\Gamma(-u,-v,-w)\}
\end{aligned}
$$

for all $u \in V$. Hence by Theorem 4.11, there exists a unique quartic mapping $Q_{2}: V \rightarrow X_{\rho}$ such that

$$
\rho\left(f_{\mathrm{q}_{2}}(u)-\mathrm{Q}_{2}(u)\right) \leqslant \frac{1}{2}\left\{\sum_{i=0}^{\infty}\left(\frac{\Gamma\left(2^{i} u, 2^{i} u,-2^{i} u\right)}{2^{4 i}}+\frac{\Gamma\left(-2^{i} u,-2^{i} u, 2^{i} u\right)}{2^{4 i}}\right)\right\}
$$


for all $u \in V$. Again $f_{o}(u)=\frac{1}{2}\{f(u)-f(-u)\}$ for all $u \in V$. Then $f_{c}(0)=0, f_{o}(u)=-f_{o}(-u)$. Hence

$$
\begin{aligned}
\rho\left(\operatorname{Df}_{\mathrm{o}}(u, v, w)\right) & =\frac{1}{2}\{\rho(\operatorname{Df}(u, v, w)+\operatorname{Df}(u, v, w))\} \\
& \leqslant \frac{1}{2}\{\rho(\operatorname{Df}(u, v, w))+\rho(\operatorname{Df}(-u,-v,-w))\} \leqslant \frac{1}{2}\{\Gamma(u, v, w)+\Gamma(-u,-v,-w)\}
\end{aligned}
$$

for all $u \in V$. Hence by Theorem 4.1, there exists a unique additive mapping $A: V \rightarrow X_{\rho}$ such that

$$
\rho\left(f_{o}(u)-A(u)\right) \leqslant \frac{1}{2}\left\{\sum_{i=0}^{\infty}\left(\frac{\Gamma\left(2^{i} u, 2^{i} u,-2^{i} u\right)}{2^{i}}+\frac{\Gamma\left(-2^{i} u,-2^{i} u, 2^{i} u\right)}{2^{i}}\right)\right\}
$$

for all $u \in V$. Since $f(u)=f_{q_{2}}(u)+f_{o}(u)$, it follows from (4.23) and (4.24) that

$$
\begin{aligned}
\rho\left(f(u)-A(u)-Q_{2}(u)\right)= & \rho\left(f_{o}(u)+f_{q_{2}}(u)-A(u)-Q_{2}(u)\right) \\
\leqslant & \rho\left(f_{q_{2}}(u)-Q_{2}(u)\right)+\rho\left(f_{o}(u)-A(u)\right) \\
\leqslant & \frac{1}{2}\left\{\sum_{i=0}^{\infty}\left(\frac{\Gamma\left(2^{i} u, 2^{i} u,-2^{i} u\right)}{2^{i}}+\frac{\Gamma\left(-2^{i} u,-2^{i} u,-2^{i} u\right)}{2^{i}}\right)\right. \\
& \left.+\sum_{i=0}^{\infty}\left(\frac{\Gamma\left(2^{i} u, 2^{i} u,-2^{i} u\right)}{2^{4 i}}+\frac{\Gamma\left(-2^{i} u,-2^{i} u, 2^{i} u\right)}{2^{4 i}}\right)\right\}
\end{aligned}
$$

for all $u \in V$. Hence we complete the proof.

Corollary 4.22. Let $\Theta$ and $\tau$ be nonnegative real numbers. If a mapping $\mathrm{f}: \mathrm{V} \rightarrow \mathrm{X}_{\rho}$ satisfies the inequality

$$
\rho(\operatorname{Df}(u, v, w)) \leqslant \Theta
$$

for all $\mathrm{u}, v, w \in \mathrm{V}$, then there exists a unique additive mapping $\mathrm{A}: \mathrm{V} \rightarrow \mathrm{X}_{\rho}$ and a unique quartic mapping $\mathrm{Q}_{2}: \mathrm{V} \rightarrow \mathrm{X}_{\rho}$ such that

$$
\rho\left(f(u)-A(u)-Q_{2}(u)\right) \leqslant \frac{16 \Theta}{15}
$$

for all $u \in V$.

Corollary 4.23. Let $\Theta$ and $\tau$ be nonnegative real numbers with $\tau \neq 1,4$. If a mapping $f: V \rightarrow X_{\rho}$ satisfies the inequality

$$
\rho(\operatorname{Df}(u, v, w)) \leqslant \Theta\left(\|u\|^{\tau}+\|v\|^{\tau}+\|w\|^{\tau}\right)
$$

for all $u, v, w \in \mathrm{V}$, then there exists a unique additive mapping $\mathrm{A}: \mathrm{V} \rightarrow \mathrm{X}_{\rho}$ and a unique quartic mapping $\mathrm{Q}_{2}: \mathrm{V} \rightarrow \mathrm{X}_{\rho}$ such that

$$
\rho\left(f(u)-A(u)-Q_{2}(u)\right) \leqslant 3 \Theta\left(\frac{1}{\left|2-2^{\tau}\right|}+\frac{1}{\left|2^{4}-2^{\tau}\right|}\right)\|u\|^{\tau}
$$

for all $\mathrm{u} \in \mathrm{V}$.

Corollary 4.24. Let $\Theta$ and $\tau$ be nonnegative real numbers with $\tau \neq \frac{1}{3}$, $\frac{4}{3}$. If a mapping $f: V \rightarrow X_{\rho}$ satisfies the inequality

$$
\rho(\operatorname{Df}(u, v, w)) \leqslant \Theta\left(\|u\|^{\tau}\|v\|^{\tau}\|w\|^{\tau}\right)
$$

for all $\mathrm{u}, v, w \in \mathrm{V}$, then there exists a unique additive mapping $\mathrm{A}: \mathrm{V} \rightarrow \mathrm{X}_{\rho}$ and a unique quartic mapping $\mathrm{Q}_{2}: \mathrm{V} \rightarrow \mathrm{X}_{\rho}$ such that

$$
\rho\left(f(u)-A(u)-Q_{2}(u)\right) \leqslant \Theta\left(\frac{1}{\left|2-2^{3 \tau}\right|}+\frac{1}{\left|2^{4}-2^{3 \tau}\right|}\right)\|u\|^{3 \tau}
$$

for all $\mathrm{u} \in \mathrm{V}$. 
Corollary 4.25. Let $\Theta$ and $\tau$ be nonnegative real numbers with $\tau \neq \frac{1}{3}, \frac{4}{3}$. If a mapping $\mathrm{f}: \mathrm{V} \rightarrow \mathrm{X}_{\rho}$ satisfies the inequality

$$
\rho(\operatorname{Df}(u, v, w)) \leqslant \Theta\left(\|u\|^{\tau}\|v\|^{\tau}\|w\|^{\tau}+\|u\|^{3 \tau}+\|v\|^{3 \tau}+\|w\|^{3 \tau}\right)
$$

for all $u, v, w \in V$, then there exists a unique additive mapping $\mathrm{A}: \mathrm{V} \rightarrow \mathrm{X}_{\rho}$ and a unique quartic mapping $\mathrm{Q}_{2}: \mathrm{V} \rightarrow \mathrm{X}_{\rho}$ such that

$$
\rho\left(f(u)-A(u)-Q_{2}(u)\right) \leqslant 4 \Theta\left(\frac{1}{\left|2-2^{3 \tau}\right|}+\frac{1}{\left|2^{4}-2^{3 \tau}\right|}\right)\|u\|^{3 \tau}
$$

for all $\mathrm{u} \in \mathrm{V}$.

4.6. Additive-quartic stability of (1.3) in modular space with $\Delta_{2}$-conditions

In this subsection, we present the Ulam-Hyers stability of the additive-quartic mixed type functional equation (1.3) using Hyers' direct method in modular space with $\Delta_{2}$-conditions.

Theorem 4.26. Let $\mathrm{j} \in\{-1,1\}$. Let $\Gamma: \mathrm{V} \rightarrow[0, \infty)$ be a function satisfying (4.14) and (4.20) for all $\mathrm{u}, v, w \in \mathrm{V}$. Let $\mathrm{f}: \mathrm{V} \rightarrow \mathrm{X}_{\rho}$ be a mapping satisfying the inequality

$$
\rho(\mathrm{D} f(u, v, w)) \leqslant \Gamma(u, v, w)
$$

for all $\mathrm{u}, v, w \in \mathrm{V}$. Then there exists a unique additive mapping $\mathrm{A}: \mathrm{V} \rightarrow \mathrm{X}_{\rho}$ and a unique quartic mapping $\mathrm{Q}_{2}: \mathrm{V} \rightarrow \mathrm{X}_{\rho}$ such that

$$
\rho\left(f(u)-A(u)-Q_{2}(u)\right) \leqslant \frac{1}{2}\left\{\sum_{i=\frac{1-j}{2}}^{\infty}\left(\frac{k^{2}}{2}\right)^{i} \Gamma\left(\frac{u}{2^{i j}}, \frac{u}{2^{i j}}, \frac{-u}{2^{i j}}\right)+\sum_{i=\frac{1-j}{2}}^{\infty}\left(\frac{k^{8}}{2^{4}}\right)^{i} \Gamma\left(\frac{u}{2^{i j}}, \frac{u}{2^{i j}}, \frac{-u}{2^{i j}}\right)\right\}
$$

for all $\mathrm{u} \in \mathrm{V}$. The mappings $\mathrm{A}(\mathrm{u})$ and $\mathrm{Q}_{2}(\mathrm{u})$ respectively are defined in (4.17) and (4.22).

Proof. Let $\mathrm{f}_{\mathrm{q}_{2}}(\mathrm{u})=\frac{1}{2}\{f(u)+f(-u)\}$ for all $\mathrm{u} \in \mathrm{V}$. Then $\mathrm{f}_{\mathrm{q}_{2}}(0)=0, \mathrm{f}_{\mathrm{q}_{2}}(\mathrm{u})=\mathrm{f}_{\mathrm{q}_{2}}(-\mathrm{u})$. Hence

$$
\begin{aligned}
& \rho\left(\operatorname{Df}_{\mathrm{q}_{2}}(\mathrm{u}, v, w)\right)=\frac{1}{2}\{\rho(\operatorname{Df}(u, v, w)+\operatorname{Df}(-u,-v,-w))\} \\
& \leqslant \frac{1}{2}\{\rho(\operatorname{Df}(u, v, w))+\rho(\operatorname{Df}(-\mathfrak{u},-v,-w))\} \leqslant \frac{1}{2}\{\Gamma(u, v, w)+\Gamma(-\mathfrak{u},-v,-w)\}
\end{aligned}
$$

for all $u \in V$. Hence by Theorem 4.16, there exist sa unique quartic mapping $Q_{2}: V \rightarrow X_{\rho}$ such that

$$
\rho\left(f_{q_{2}}(u)-Q_{2}(u)\right) \leqslant \frac{1}{2}\left\{\sum_{i=0}^{\infty}\left(\frac{k^{8}}{16}\right)^{i}\left(\Gamma\left(\frac{u}{2^{i}}, \frac{u}{2^{i}}, \frac{-u}{2^{i}}\right)+\Gamma\left(\frac{-u}{2^{i}}, \frac{-u}{2^{i}}, \frac{u}{2^{i}}\right)\right)\right\}
$$

for all $u \in V$. Again $f_{o}(u)=\frac{1}{2}\{f(u)-f(-u)\}$ for all $u \in V$. Then $f_{c}(0)=0, f_{o}(u)=-f_{o}(-u)$. Hence

$$
\begin{aligned}
\rho\left(\operatorname{Df}_{\mathrm{o}}(u, v, w)\right) & =\frac{1}{2}\{\rho(\operatorname{Df}(u, v, w)+\operatorname{Df}(u, v, w))\} \\
& \leqslant \frac{1}{2}\{\rho(\operatorname{Df}(u, v, w))+\rho(\operatorname{Df}(-u,-v,-w))\} \leqslant \frac{1}{2}\{\Gamma(u, v, w)+\Gamma(-u,-v,-w)\}
\end{aligned}
$$

for all $u \in V$. Hence by Theorem 4.6, there exists a unique additive mapping $A: V \rightarrow X_{\rho}$ such that

$$
\rho\left(f_{o}(u)-A(u)\right) \leqslant \frac{1}{2}\left\{\sum_{i=0}^{\infty}\left(\frac{k^{2}}{2}\right)^{i}\left(\Gamma\left(\frac{u}{2^{i}}, \frac{u}{2^{i}}, \frac{-u}{2^{i}}\right)+\Gamma\left(\frac{-u}{2^{i}}, \frac{-u}{2^{i}}, \frac{u}{2^{i}}\right)\right)\right\}
$$


for all $u \in V$. Since $f(u)=f_{q_{2}}(u)+f_{o}(u)$, it follows from (4.25) and (4.26) that

$$
\begin{aligned}
\rho\left(f(u)-A(u)-Q_{2}(u)\right)= & \rho\left(f_{o}(u)+f_{q_{2}}(u)-A(u)-Q_{2}(u)\right) \\
\leqslant & \rho\left(f_{q_{2}}(u)-Q_{2}(u)\right)+\rho\left(f_{o}(u)-A(u)\right) \\
\leqslant & \frac{1}{2}\left\{\sum_{i=0}^{\infty}\left(\frac{k^{8}}{16}\right)^{i}\left(\Gamma\left(\frac{u}{2^{i}}, \frac{u}{2^{i}}, \frac{-u}{2^{i}}\right)+\Gamma\left(\frac{-u}{2^{i}}, \frac{-u}{2^{i}}, \frac{u}{2^{i}}\right)\right)\right. \\
& \left.+\sum_{i=0}^{\infty}\left(\frac{k^{2}}{2}\right)^{i}\left(\Gamma\left(\frac{u}{2^{i}}, \frac{u}{2^{i}}, \frac{-u}{2^{i}}\right)+\Gamma\left(\frac{-u}{2^{i}}, \frac{-u}{2^{i}}, \frac{u}{2^{i}}\right)\right)\right\}
\end{aligned}
$$

for all $u \in V$. Hence we complete the proof.

Corollary 4.27. Let $\Theta$ and $\tau$ be nonnegative real numbers and $\mathrm{X}_{\rho}$ satisfies $\Delta_{2}$ condition. If a mapping $f: \mathrm{V} \rightarrow \mathrm{X}_{\rho}$ satisfies the inequality

$$
\rho(\operatorname{Df}(u, v, w)) \leqslant \Theta
$$

for all $\mathrm{u}, v, w \in \mathrm{V}$, then there exists a unique additive mapping $\mathrm{A}: \mathrm{V} \rightarrow \mathrm{X}_{\rho}$ and a unique quartic mapping $\mathrm{Q}_{2}: \mathrm{V} \rightarrow \mathrm{X}_{\rho}$ such that

$$
\rho\left(f(u)-A(u)-Q_{2}(u)\right) \leqslant k^{2} \Theta\left(1+\frac{k^{6}}{15}\right)
$$

for all $\mathrm{u} \in \mathrm{V}$.

Corollary 4.28. Let $\Theta$ and $\tau$ be nonnegative real numbers and $X_{\rho}$ satisfies $\Delta_{2}$ condition with $2^{\tau} \neq k^{2}, k^{8}$. If a mapping $\mathrm{f}: \mathrm{V} \rightarrow \mathrm{X}_{\rho}$ satisfies the inequality

$$
\rho(\operatorname{Df}(u, v, w)) \leqslant \Theta\left(\|u\|^{\tau}+\|v\|^{\tau}+\|w\|^{\tau}\right)
$$

for all $u, v, w \in \mathrm{V}$, then there exists a unique additive mapping $\mathrm{A}: \mathrm{V} \rightarrow \mathrm{X}_{\rho}$ and a unique quartic mapping $\mathrm{Q}_{2}: \mathrm{V} \rightarrow \mathrm{X}_{\rho}$ such that

$$
\rho\left(f(u)-A(u)-Q_{2}(u)\right) \leqslant 3 \Theta\left(\frac{k^{2}}{\left|k^{2}-2^{\tau}\right|}+\frac{k^{8}}{\left|k^{8}-2^{\tau}\right|}\right)\|u\|^{\tau}
$$

for all $\mathrm{u} \in \mathrm{V}$.

Corollary 4.29. Let $\Theta$ and $\tau$ be nonnegative real numbers and $X_{\rho}$ satisfies $\Delta_{2}$ condition with $2^{3 \tau} \neq k^{2}, k^{8}$. If a mapping $\mathrm{f}: \mathrm{V} \rightarrow \mathrm{X}_{\rho}$ satisfies the inequality

$$
\rho(\operatorname{Df}(u, v, w)) \leqslant \Theta\left(\|u\|^{\tau}\|v\|^{\tau}\|w\|^{\tau}\right)
$$

for all $u, v, w \in \mathrm{V}$, then there exists a unique additive mapping $\mathrm{A}: \mathrm{V} \rightarrow \mathrm{X}_{\rho}$ and a unique quartic mapping $\mathrm{Q}_{2}: \mathrm{V} \rightarrow \mathrm{X}_{\rho}$ such that

$$
\rho\left(f(u)-A(u)-Q_{2}(u)\right) \leqslant \Theta\left(\frac{k^{2}}{\left|k^{2}-2^{3 \tau}\right|}+\frac{k^{8}}{\left|k^{8}-2^{3 \tau}\right|}\right)\|u\|^{3 \tau}
$$

for all $\mathrm{u} \in \mathrm{V}$.

Corollary 4.30. Let $\Theta$ and $\tau$ be nonnegative real numbers and $X_{\rho}$ satisfies $\Delta_{2}$ condition with $2^{3 \tau} \neq k^{2}, k^{8}$. If a mapping $\mathrm{f}: \mathrm{V} \rightarrow \mathrm{X}_{\rho}$ satisfies the inequality

$$
\rho(\operatorname{Df}(u, v, w)) \leqslant \Theta\left(\|u\|^{\tau}\|v\|^{\tau}\|w\|^{\tau}+\|u\|^{3 \tau}+\|v\|^{3 \tau}+\|w\|^{3 \tau}\right)
$$

for all $\mathrm{u}, v, w \in \mathrm{V}$, then there exists a unique additive mapping $\mathrm{A}: \mathrm{V} \rightarrow \mathrm{X}_{\rho}$ and a unique quartic mapping $\mathrm{Q}_{2}: \mathrm{V} \rightarrow \mathrm{X}_{\rho}$ such that

$$
\rho\left(f(u)-A(u)-Q_{2}(u)\right) \leqslant 4 \Theta\left(\frac{k^{2}}{\left|k^{2}-2^{3 \tau}\right|}+\frac{k^{8}}{\left|k^{8}-2^{3 \tau}\right|}\right)\|u\|^{3 \tau}
$$

for all $\mathrm{u} \in \mathrm{V}$. 


\section{Conclusion}

In this article, we have proved the stability results of additive functional equation, quartic functional equation, and additive-quartic mixed type functional equations in modular spaces with and without using the $\Delta_{2}$-condition by the direct method.

\section{References}

[1] J. Aczél, J. Dhombres, Functional Equations in Several Variables, Cambridge University Press, Cambridge, (1989).

[2] T. Aoki, On the stability of the linear transformation in Banach spaces, J. Math. Soc. Japan, 2 (1950), 64-66. 1

[3] D. G. Bourgin, Classes of transformations and bordering transformations, Bull. Amer. Math. Soc., 57 (1951), $223-237$.

[4] Y. Cho, M. B. Ghaemi, M. Choubin, M. Eshaghi Gordji, On the Hyers-Ulam stability of sextic functional equations in b-homogeneous probabilistic modular spaces, Math. Inequal. Appl., 16 (2013), 1097-1114. 1

[5] S. Czerwik, On the stability of the quadratic mapping in normed spaces, Abh. Math. Sem. Univ. Hamburg, 62 (1992), 59-64.

[6] S. Czerwik, Functional Equations and Inequalities in Several Variables, World Scientific Publishing Co., River Edge, (2002).

[7] E. Elqorachi, M. T. Rassias, Generalized Hyers-Ulam Stability of Trigonometric Functional Equations, Mathematics, 6 (2018), 11 pages.

[8] Z. Gajda, On stability of additive mappings, Internat. J. Math. Math. Sci., 14 (1991), 431-434.

[9] P. Găvruta, A generalization of the Hyers-Ulam-Rassias stability of approximately additive mappings, J. Math. Anal. Appl., 184 (1994), 431-436. 1

[10] M. Heydari, S. M. Hosseini, G. B. Loghmani, Numerical solution of singular IVPs of Lane-Emden type using integral operator and radial basis functions, Int. J. Ind. Math., 4 (2012), 135-146. 1

[11] D. H. Hyers, On the stability of the linear functional equation, Proc. Nat. Acad. Sci. U.S.A., 27 (1941), 222-224. 1

[12] D. H. Hyers, G. Isac, T. M. Rassias, Stability of Functional Equations in Several Variables, Birkäuser, Boston, (1998).

[13] D. H. Hyers, T. M. Rassias, Approximate homomorphisms, Aequationes Math., 44 (1992), 125-153.

[14] Y. F. Jin, C. Park, M. T. Rassias , Hom-derivations in C*-ternary algebras, Acta Math. Sin. (Engl. Ser.), 36 (2020), 1025-1038.

[15] S.-M. Jung, Hyers-Ulam stability of a system of first order linear differential equations with constant coefficients, J. Math. Anal. Appl., 320 (2006), 549-561.

[16] S.-M. Jung, Hyers-Ulam-Rassias Stability of Functional Equations in Nonlinear Analysis, Springer, New York, (2011).

[17] S.-M. Jung, K. S. Lee, M. T. Rassias, S. M. Yang, Approximation Properties of Solutions of a Mean Value-Type Functional Inequality, II, Mathematics, 8 (2020), 8 pages.

[18] S.-M. Jung, D. Popa, M. T. Rassias, On the stability of the linear functional equation in a single variable on complete metric groups, J. Global Optim., 59 (2014), 165-171.

[19] S.-M. Jung, M. T. Rassias , A linear functional equation of third order associated to the Fibonacci numbers, Abstr. Appl. Anal., 2014 (2014), 8 pages.

[20] S.-M. Jung, M. T. Rassias, C. Mortici, On a functional equation of trigonometric type, Appl. Math. Comput., 252 (2015), 294-303.

[21] P. Kannappan, Functional Equations and Inequalities with Applications, Springer, New York, (2009).

[22] H.-M. Kim, H.-Y. Shin, Refined stability of additive and quadratic functional equations in modular spaces, J. Inequal. Appl., 2017 (2017), 13 pages. 1

[23] Y.-H. Lee, S.-M. Jung, M. T. Rassias, On an n-dimensional mixed type additive and quadratic functional equation, Appl. Math. Comput., 228 (2014), 13-16.

[24] Y.-H. Lee, S.-M. Jung, M. T. Rassias, Uniqueness theorems on functional inequalities concerning cubic-quadratic-additive equation, J. Math. Inequal., 12 (2018), 43-61.

[25] W. A. J. Luxemburg, Banach Function Spaces, Ph.D. Thesis (Delft University of Technology), Delft, (1955). 2

[26] S. Mazur, W. Orlicz, On some classes of linear spaces, Studia Math., 17 (1958), 97-119. 2

[27] T. Miura, S. Miyajima, S. E. Takahasi, A characterization of Hyers-Ulam stability of first order linear differential operators, J. Math. Anal. Appl., 286 (2003), 136-146.

[28] C. Mortici, M. T. Rassias, S.-M. Jung, On the stability of a functional equation associated with the Fibonacci numbers, Abstr. Appl. Anal., 2014 (2014), 6 pages.

[29] J. Musielak, W. Orlicz, On modular spaces, Studia Math., 18 (1959), 591-597. 2

[30] J. Musielak, W. Orlicz, Some remarks on modular spaces, Bull. Acad. Polon. Sci. Ser. Sci. Math. Astr. Phys., 7 (1959), 661-668. 2

[31] H. Nakano, Modulared Semi-Ordered Linear Spaces, Maruzen Co., Tokyo, (1950). 2

[32] C. Park, A. Bodaghi, S. O. Kim, A fixed point approach to stability of additive mappings in modular spaces without $\Delta_{2}$-conditions, J. Comput. Anal. Appl., 24 (2018), 1038-1048. 1

[33] C. Park, M. T. Rassias, Additive functional equations and partial multipliers in $\mathrm{C}^{*}$-algebras, Rev. R. Acad. Cienc. Exactas Fis. Nat. Ser. A Mat. RACSAM, 113 (2019), 2261-2275. 
[34] C. Park, J. M. Rassias, A. Bodaghi, S. O. Kim, Approximate homomorphisms from ternary semigroups to modular spaces, Rev. R. Acad. Cienc. Exactas Fís. Nat. Ser. A Mat. RACSAM, 113 (2019), 2175-2188. 1

[35] M. Ramdossa, D. Pachaiyappana, H. Duttab, Functional equation and its modular stability with and without $\Delta_{p}$ condition, Filomat, 34 (2020), 919-930. 1

[36] T. M. Rassias, On the stability of linear mappings in Banach spaces, Proc. Amer. Math. Soc., 72 (1978), 297-300. 1

[37] J. M. Rassias, On approximation of approximately linear mappings by linear mappings, Bull. Sci. Math. (2), 108 (1984), 445-446. 1

[38] T. M. Rassias, Functional Equations and Inequalities, Springer Science \& Business Media, Berlin (2000).

[39] T. M. Rassias, Solution of a functional equation problem of Steven Butler, Octogon Math. Mag., 12 (2004), 152-153.

[40] K. Ravi, M. Arunkumar, J. M. Rassias, Ulam stability for the orthogonally general Euler-Lagrange type functional equation, Int. J. Math. Stat., 3 (2008), 36-46. 1

[41] P. K. Sahoo, P. Kannappan, Introduction to Functional Equations, CRC Press, Boca Raton, (2011).

[42] T. Trif, On the stability of a functional equation deriving from an inequality of Popoviciu for convex functions, J. Math. Anal. Appl., 272 (2002), 604-616.

[43] S. M. Ulam, Problems in Modern Mathematics, Science Editions John Wiley \& Sons, New York, (1964). 1

[44] K. Wongkum, P. Chaipunya, P. Kumam, On the generalized Ulam-Hyers-Rassias stability of quadratic mappings in modular spaces without $\Delta_{2}$-conditions, J. Funct. Spaces, 2015 (2015), 6 pages. 1

[45] K. Wongkum, P. Kumam, Y. J. Cho, P. Thounthong, P. Chaipunya, On the generalized Ulam-Hyers-Rassias stability for quartic functional equation in modular spaces, J. Nonlinear Sci. Appl., 10 (2017), 1399-1406. 1 